International Journal of Pure and Applied Mathematics

Volume $97 \quad$ No. $4 \quad 2014,511-514$

ISSN: 1311-8080 (printed version); ISSN: 1314-3395 (on-line version)

url: http://www.ijpam.eu

doi: http://dx.doi.org/10.12732/ijpam.v97i4.10

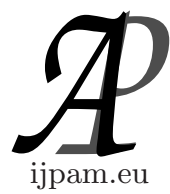

\title{
AN IMPROVED BINOMIAL APPROXIMATION FOR THE BETA BINOMIAL DISTRIBUTION
}

\author{
K. Teerapabolarn \\ Department of Mathematics \\ Faculty of Science \\ Burapha University \\ Chonburi, 20131, THAILAND
}

\begin{abstract}
This paper gives an approximation of the beta binomial distribution with parameters $n, \alpha$ and $\beta$ by an improved binomial distribution with parameters $n$ and $\frac{\alpha}{\alpha+\beta}$. The improved binomial approximation is more accurate than the binomial approximation when $\alpha+\beta$ is large.
\end{abstract}

AMS Subject Classification: 62E17, 60F05

Key Words: beta binomial distribution, binomial distribution, improved binomial distribution, probability function

\section{Introduction}

The binomial distribution with parameters $n$ and $p$ can be thought of as the distribution of the number of successes that occur in $n$ independent Bernoulli trials with the probability of success in each trial is $p$. If $p$ is a random variable that has a beta distribution with shape parameters $\alpha$ and $\beta$, then the resulting distribution is referred to as the beta binomial distribution with parameters $n$,

Received: July 18, 2014

(c) 2014 Academic Publications, Ltd. url: www.acadpubl.eu 
$\alpha$ and $\beta$, where $\alpha, \beta \in \mathbb{R}^{+}$and $n \in \mathbb{N}$. Let $X$ be the beta binomial random variable with the probability function as follows:

$$
\mathbf{b b}_{n, \alpha, \beta}(x)=\left(\begin{array}{l}
n \\
x
\end{array}\right) \frac{\Gamma(\alpha+x) \Gamma(\beta+n-x) \Gamma(\alpha+\beta)}{\Gamma(\alpha+\beta+n) \Gamma(\alpha) \Gamma(\beta)}, x=0,1, \ldots, n .
$$

The mean and variance of $X$ are $\mu=\frac{n \alpha}{\alpha+\beta}$ and $\sigma^{2}=\frac{n \alpha \beta(n+\alpha+\beta)}{(\alpha+\beta)^{2}(1+\alpha+\beta)}$, respectively. Because the beta binomial distribution is obtained from a binomial distribution, it is natural to speculate that the beta binomial distribution can be approximated by the binomial distribution. In this case, [2] gave a bound on the total variation distance between two such distributions. In this paper, we give an improved binomial probability function, $\widehat{\mathbf{b}}_{n, p}(x)$, to approximate the beta binomial probability function. The accuracy of the approximation is measured in the form of $\left|\mathbf{b b}_{n, \alpha, \beta}(x)-\widehat{\mathbf{b}}_{n, p}(x)\right|$ for $x \in\{0,1, \ldots, n\}$, which is in Section 2. In Section 3, some numerical examples have been given to illustrate the improved approximation and the conclusion of this study is presented in the last section.

\section{Result}

Applying the property in [1], the following lemma is also obtained.

Lemma 2.1. For $\alpha, \beta \in \mathbb{R}^{+}, x \in \mathbb{N}$ and $0<p<1$, then

$$
\begin{aligned}
& \prod_{i=0}^{x-1}\left(p+\frac{i}{\alpha+\beta}\right)=p^{x}\left[1+\frac{x(x-1)}{2(\alpha+\beta) p}\right]+O\left(\frac{1}{(\alpha+\beta)^{2}}\right), \\
& \prod_{i=0}^{x-1}\left(1+\frac{i}{\alpha+\beta}\right)=1+\frac{x(x-1)}{2(\alpha+\beta)}+O\left(\frac{1}{(\alpha+\beta)^{2}}\right) .
\end{aligned}
$$

Theorem 2.1. Let $\mathbf{b}_{n, p}(x)=\left(\begin{array}{l}n \\ x\end{array}\right) p^{x}(1-p)^{n-x}$ for $x \in\{0,1, \ldots, n\}$ and $p=\frac{\alpha}{\alpha+\beta}$. Then we have the following:

$$
\mathbf{b b}_{n, \alpha, \beta}(x)=\widehat{\mathbf{b}}_{n, p}(x)+O\left(\frac{1}{(\alpha+\beta)^{2}}\right)
$$

and for large $\alpha+\beta$,

$$
\mathbf{b b}_{n, \alpha, \beta}(x) \approx \widehat{\mathbf{b}}_{n, p}(x),
$$


where $\widehat{\mathbf{b}}_{n, p}(x)=\mathbf{b}_{n, p}(x)\left\{1+\frac{(n-x)(n-x-1)}{2 \beta}+\frac{x(x-1)}{2 \alpha}\right\} /\left\{1+\frac{n(n-1)}{2(\alpha+\beta)}\right\}$.

Proof. Let $\delta=\left\{\begin{array}{ll}0 & \text { if } x=0, \\ x-1 & \text { if } x=1, \ldots, n .\end{array}\right.$ Applying Lemma 2.1, it follows that

$$
\begin{aligned}
\mathbf{b b}_{n, \alpha, \beta}(x)= & \left(\begin{array}{l}
n \\
x
\end{array}\right) \frac{[(\alpha+\delta) \cdots \alpha][(\beta+n-x-1) \cdots \beta]}{(\alpha+\beta+n-1) \cdots(\alpha+\beta)} \\
= & \left(\begin{array}{l}
n \\
x
\end{array}\right) \frac{\prod_{i=0}^{\delta}\left(p+\frac{i}{\alpha+\beta}\right) \prod_{i=0}^{n-x-1}\left(1-p+\frac{i}{\alpha+\beta}\right)}{\prod_{i=0}^{n-1}\left(1+\frac{i}{\alpha+\beta}\right)} \\
= & \left(\begin{array}{l}
n \\
x
\end{array}\right) \frac{p^{x}(1-p)^{n-x}}{1+\frac{n(n-1)}{2(\alpha+\beta)}}\left\{1+\frac{(n-x)(n-x-1)}{2 \beta}+\frac{x(x-1)}{2 \alpha}\right\} \\
& +O\left(\frac{1}{(\alpha+\beta)^{2}}\right) \\
= & \frac{\mathbf{b}_{n, p}(x)}{1+\frac{n(n-1)}{2(\alpha+\beta)}}\left\{1+\frac{(n-x)(n-x-1)}{2 \beta}+\frac{x(x-1)}{2 \alpha}\right\}+O\left(\frac{1}{(\alpha+\beta)^{2}}\right) \\
= & \widehat{\mathbf{b}}_{n, p}(x)+O\left(\frac{1}{(\alpha+\beta)^{2}}\right) .
\end{aligned}
$$

If $\alpha+\beta$ is large, then $O\left(\frac{1}{(\alpha+\beta)^{2}}\right) \approx 0$. Hence $\mathbf{b b}_{n, \alpha, \beta}(x) \approx \widehat{\mathbf{b}}_{n, p}(x)$.

\section{Numerical Examples}

The following examples are given to illustrate how well an improved binomial distribution approximates a beta binomial distribution.

3.1. Let $n=10, \alpha=10$ and $\beta=90$, then $p=\frac{10}{100}$ and the numerical results are as follows:

\begin{tabular}{cccc|c|c}
\hline$x$ & $\mathbf{b b}_{n, \alpha, \beta}(x)$ & $\widehat{\mathbf{b}}_{n, p}(x)$ & $\mathbf{b}_{n, p}(x)$ & $\mathbf{b b}_{n, \alpha, \beta}(x)-\widehat{\mathbf{b}}_{n, p}(x)$ & $\left|\mathbf{b b}_{n, \alpha, \beta}(x)-\mathbf{b}_{n, p}(x)\right|$ \\
\hline 0 & 0.36541727 & 0.36070183 & 0.34867844 & 0.00471544 & 0.01673883 \\
1 & 0.36910835 & 0.37406116 & 0.38742049 & 0.00495281 & 0.01831214 \\
2 & 0.18643738 & 0.18851495 & 0.19371024 & 0.00207757 & 0.00727286 \\
3 & 0.06150512 & 0.06069423 & 0.05739563 & 0.00081089 & 0.00410949 \\
4 & 0.01457543 & 0.01359756 & 0.01116026 & 0.00097787 & 0.00341517 \\
5 & 0.00257755 & 0.00216649 & 0.00148803 & 0.00041106 & 0.00108952 \\
6 & 0.00034276 & 0.00024389 & 0.00013778 & 0.00009887 & 0.00020498 \\
7 & 0.00003370 & 0.00001890 & 0.00000875 & 0.00001479 & 0.00002495 \\
8 & 0.00000233 & 0.00000096 & 0.00000036 & 0.00000138 & 0.00000197 \\
9 & 0.00000010 & 0.00000003 & 0.00000001 & 0.00000007 & 0.00000009 \\
\hline
\end{tabular}


3.2. Let $n=30, \alpha=30$ and $\beta=470$, then $p=\frac{30}{500}$ and the numerical results are as follows:

\begin{tabular}{cccc|c|c}
\hline$x$ & $\mathbf{b b}_{n, \alpha, \beta}(x)$ & $\widehat{\mathbf{b}}_{n, p}(x)$ & $\mathbf{b}_{n, p}(x)$ & $\mathbf{b b}_{n, \alpha, \beta}(x)-\widehat{\mathbf{b}}_{n, p}(x)$ & $\left|\mathbf{b b}_{n, \alpha, \beta}(x)-\mathbf{b}_{n, p}(x)\right|$ \\
\hline 0 & 0.16482252 & 0.16089581 & 0.15625561 & 0.00392672 & 0.00856692 \\
1 & 0.29727509 & 0.29822559 & 0.29921286 & 0.00095050 & 0.00193777 \\
2 & 0.26832360 & 0.27213121 & 0.27693105 & 0.00380761 & 0.00860745 \\
3 & 0.16124611 & 0.16293414 & 0.16498020 & 0.00168803 & 0.00373410 \\
4 & 0.07241446 & 0.07189874 & 0.07108190 & 0.00051571 & 0.00133256 \\
5 & 0.02586440 & 0.02487539 & 0.02359314 & 0.00098901 & 0.00227126 \\
6 & 0.00763541 & 0.00700370 & 0.00627477 & 0.00063171 & 0.00136064 \\
7 & 0.00191162 & 0.00164366 & 0.00137320 & 0.00026796 & 0.00053842 \\
8 & 0.00041331 & 0.00032676 & 0.00025200 & 0.00008655 & 0.00016131 \\
9 & 0.00007819 & 0.00005565 & 0.00003932 & 0.00002254 & 0.00003887 \\
10 & 0.00001307 & 0.00000819 & 0.00000527 & 0.00000488 & 0.00000780 \\
11 & 0.00000194 & 0.00000105 & 0.00000061 & 0.00000090 & 0.00000133 \\
12 & 0.00000026 & 0.00000012 & 0.00000006 & 0.00000014 & 0.00000020 \\
13 & 0.00000003 & 0.00000001 & 0.00000001 & 0.00000002 & 0.00000003 \\
\hline
\end{tabular}

From the examples 3.1 and 3.2, it is seen that the improved binomial approximation is more accurate than the binomial approximation.

\section{Conclusion}

In this study, an improved binomial distribution with parameters $n$ and $p=\frac{\alpha}{\alpha+\beta}$ for approximating the beta binomial distribution with parameters $n, \alpha$ and $\beta$ is derived. The improvement of the binomial approximation is more appropriate than the binomial approximation, that is, the improved binomial distribution can be used as a good approximation of the beta binomial distribution when $\alpha+\beta$ is sufficiently large.

\section{References}

[1] D.P. Hu, Y.Q. Cui, A.H. Yin , An improved negative binomial approximation for negative hypergeometric distribution, Applied Mechanics and Materials, 427-429 (2013), 2549-2553.

[2] K. Teerapabolarn, A bound on the binomial approximation to the beta binomial distribution, International Mathematical Forum, 3 (2008), 13551358. 